\title{
A new methodical approach in neuroscience: assessing inter-personal brain coupling using functional near-infrared imaging (fNIRI) hyperscanning
}

\author{
Felix Scholkmann ${ }^{1,2}{ }^{*}$, Lisa Holper ${ }^{1}$, Ursula Wolf ${ }^{2}$ and Martin Wolf ${ }^{1}$ \\ Biomedical Optics Research Laboratory, Division of Neonatology, University Hospital Zurich, Zurich, Switzerland \\ ${ }^{2}$ Institute for Complementary Medicine, University of Bern, Bern, Switzerland
}

\section{Edited by:}

Leonhard Schilbach, University of

Hospital Cologne, Germany

\section{Reviewed by:}

Peter Kirsch, Zentralinstitut für

Seelische Gesundheit, Germany

Ivana Konvalinka, Technical University

of Denmark, Denmark

*Correspondence:

Felix Scholkmann, Biomedical Optics Research Laboratory, Division of

Neonatology, University Hospital

Zurich, Frauenklinikstr. 10, 8091 Zurich,

Switzerland

e-mail: Felix.Scholkmann@usz.ch
Since the first demonstration of how to simultaneously measure brain activity using functional magnetic resonance imaging (fMRI) on two subjects about 10 years ago, a new paradigm in neuroscience is emerging: measuring brain activity from two or more people simultaneously, termed "hyperscanning". The hyperscanning approach has the potential to reveal inter-personal brain mechanisms underlying interaction-mediated brain-to-brain coupling. These mechanisms are engaged during real social interactions, and cannot be captured using single-subject recordings. In particular, functional near-infrared imaging (fNIRI) hyperscanning is a promising new method, offering a cost-effective, easy to apply and reliable technology to measure inter-personal interactions in a natural context. In this short review we report on fNIRI hyperscanning studies published so far and summarize opportunities and challenges for future studies.

Keywords: functional near-infrared imaging, hyperscanning, brain-to-brain coupling, inter-personal brain activity, hyperconnectivity, social neuroscience

\section{INTRODUCTION}

A new approach to investigate neuronal correlates of interaction between two or more people is emerging: the measurement of inter-personal (between-person) dynamics of brain activity, termed "hyperscanning" (for reviews, see Astolfi et al., 2011; Dumas et al., 2011; Babiloni and Astolfi, 2012; Genvins et al., 2012; Konvalinka and Roepstorff, 2012; Schilbach et al., 2013). This approach constitutes a third stage in the development of neuroscience. The first stage comprised the classic cognitive neuroscience paradigm, i.e., the measurement of intra-personal (within-person) brain activity with a focus on the functional specialization of the individual brain as well as its activity in creating representations of the inner and outer world. The second stage emerged from the field of social neuroscience and was developed, popularized and formulized in the 1990s by Cacioppo and Berntson (1992) as a "multi-level analysis of social psychological phenomena". The main methodological approach in social neuroscience is to investigate intra-personal brain dynamics during inter-personal interactions. Research in this field revealed that specific brain structures of the "social brain" are involved in social cognition, e.g., brain areas constituting the "mirror neuron system" (Saxe, 2006; Frith, 2007), the "theory of mind" (Premack and Woodruff, 1978; Frith and Frith, 2001) or the "empathy network" (Bernhardt and Singer, 2012). Despite the impressive insights into the neurobiological aspects of human social interaction that have emerged from these two approaches, the neurobiological processes involved in real interpersonal interactions (i.e., the brain-to-brain mechanisms between persons)-which represent the "dark matter" in social neuroscience—cannot be investigated with these methodologies (Przyrembel et al., 2012). As a consequence, the next step in social neuroscience can be regarded as the assessment of the neuronal correlates of social interaction dynamics, and thus as moving from the observer's perspective towards a truly interactive approach, i.e., "a shift from a single-brain to a multi-brain frame of reference" (Hasson et al., 2012). The measurement of brain activity from two or more people simultaneously, and the quantification of the interpersonal brain-to-brain coupling is a methodological tool of particular importance in this approach, which allows the assessment of the bidirectional information flow between interacting persons. This aspect "has been largely neglected" (Hari and Kujala, 2009) in neuroimaging studies so far. The significance of this new step in social neuroscience, i.e., "two-person neuroscience" (Hari and Kujala, 2009), is evident in the growing number papers published about this topic; see for example the special issue titled "Towards a neuroscience of social interaction" published recently in this journal. Brain-to-brain coupling may serve an integral function in social interaction, as for example in a teacher-student (teachinglearning) interaction, where "interpersonal synchronization may support reciprocal, dynamical feedback between teacher and students, through implicit behavioral contagion" (Watanabe, 2013). The first studies using hyperscanning approaches can be traced back to electroencephalography (EEG) studies from the 1960s and 1970s (Duane and Behrendt, 1965; Hearne, 1977). The first hyperscanning study employing functional magnetic resonance imaging (fMRI) was conducted 11 years ago by Montague et al. (2002) who coined the term hyperscanning. Connections between two brains were termed hyperconnections and each connection a 
hyperlink (see Figure 1A). The first hyperscanning study applying functional near-infrared imaging (fNIRI) was published very recently in 2011 by Funane et al. (2011). So far this research, using EEG, fMRI and fNIRI, showed that brain-to-brain coupling is a non-local emergent phenomenon, i.e., it cannot be reduced to the local activity of a single brain (Hari and Kujala, 2009; Chatel-Goldman et al., 2013). That the "interaction process as a whole has properties that cannot be reduced to the contributions of the isolated agents" was also recently shown by an evolutionary robotics model simulating social interaction (Froese et al., 2013).

The aim of the present paper is (i) to review the fNIRI hyperscanning studies performed so far, and (ii) to summarize opportunities and challenges for future fNIRI hyperscanning studies.

\section{BEYOND INDIVIDUAL BRAIN ACTIVITY: fNIRI HYPERSCANNING}

Until spring 2013, seven research papers were published employing the fNIRI hyperscanning methodology. A comparison with the number of hyperscanning studies by other neuroimaging modalities can be found in Figure 1A. All fNIRI studies applied near-infrared spectroscopy (NIRS) devices with more than 4 channels thus enabling near-infrared imaging (NIRI), i.e., measuring changes in oxy- and deoxyhemoglobin concentration $\left(\left[\mathrm{O}_{2} \mathrm{Hb}\right]\right.$ and $[\mathrm{HHb}]$, respectively) at different locations (realized by different source-detector channels) of the heads of two subjects simultaneously. For a review on fNIRI, refer to Ferrari and Quaresima (2012) and Scholkmann et al. (2013b). Table 1 depicts the details of these studies.

Funane et al. (2011) employed a 22-channel NIRI device to measure simultaneously in two persons changes in $\left[\mathrm{O}_{2} \mathrm{Hb}\right]$ and $[\mathrm{HHb}]$ in the medial prefrontal cortex (PFC) while performing a cooperative button-press task. Two participants were instructed to synchronize their respective button presses as best as possible. Twelve subjects were measured and the $\left[\mathrm{O}_{2} \mathrm{Hb}\right]$ signals were analyzed. The authors reported an increase in the covariance ( $\mathrm{Cov}$ ) of $\left[\mathrm{O}_{2} \mathrm{Hb}\right]$ when the subjects successfully interacted in the cooperative task, i.e., when button-presses were highly synchronized. They also found a significant positive correlation between the task performance and the degree of $\left[\mathrm{O}_{2} \mathrm{Hb}\right] \mathrm{Cov}$.

Cui et al. (2012) employed also a 22-channel NIRI device to measure $\left[\mathrm{O}_{2} \mathrm{Hb}\right]$ and $[\mathrm{HHb}]$ changes in two persons simultaneously during two different tasks: a cooperation task, i.e., simultaneous button-pressing, with the aim to reach a smallest possible time difference between the responses of the two subjects, and a competition task, i.e., simultaneous button-pressing, with the aim to respond faster than the competitor. Twenty-two subjects were measured and $\left[\mathrm{O}_{2} \mathrm{Hb}\right]$ was analyzed. The brain-to-brain coupling was quantified by calculating the wavelet coherence (WC)-a measure of the cross-correlation of two time series as a function of frequency and time. The authors found that the coherence (in the frequency band $0.08-0.3 \mathrm{~Hz}$ ) between the two subjects' right superior frontal cortices increased during the cooperation, but not during the competition task.

Dommer et al. (2012) performed a fNIRI hyperscanning study with two novel 4-channel wireless NIRI-devices (Muehlemann et al., 2008), allowing an unconstrained setting without disturbing cables. Changes in $\left[\mathrm{O}_{2} \mathrm{Hb}\right]$ and $[\mathrm{HHb}]$ were recorded on the left PFC during performance on a dual n-back task simultaneously in paired players (eight subjects) as compared to single players (seven subjects). Signal analysis was performed on changes in total hemoglobin concentration $(\mathrm{tHb})\left([\mathrm{tHb}]=\left[\mathrm{O}_{2} \mathrm{Hb}\right]+[\mathrm{HHb}]\right)$. Both, the increase in the block-averaged [ $\mathrm{tHb}]$ hemodynamic response during the tasks as well as the WC were determined. It was found that (i) the hemodynamic response was larger for the paired compared to the single players, and (ii) that interpersonal brain coherence increased during the joint n-back task as compared to baseline. The coherence increase was found in the frequency bands $0.7-4 \mathrm{~Hz}$ (related to the heart rate (HR)) and $0.06-0.2 \mathrm{~Hz}$ (related to spontaneous low-frequency oscillations (LFOs)), indicating that the joint performance was associated with a synchronization of HR and LFOs.

Holper et al. (2012) investigated, with the same fNIRI-setup as Dommer et al. (2012), how brain-to-brain coupling is influenced during imitation. A paced finger-tapping task was performed by
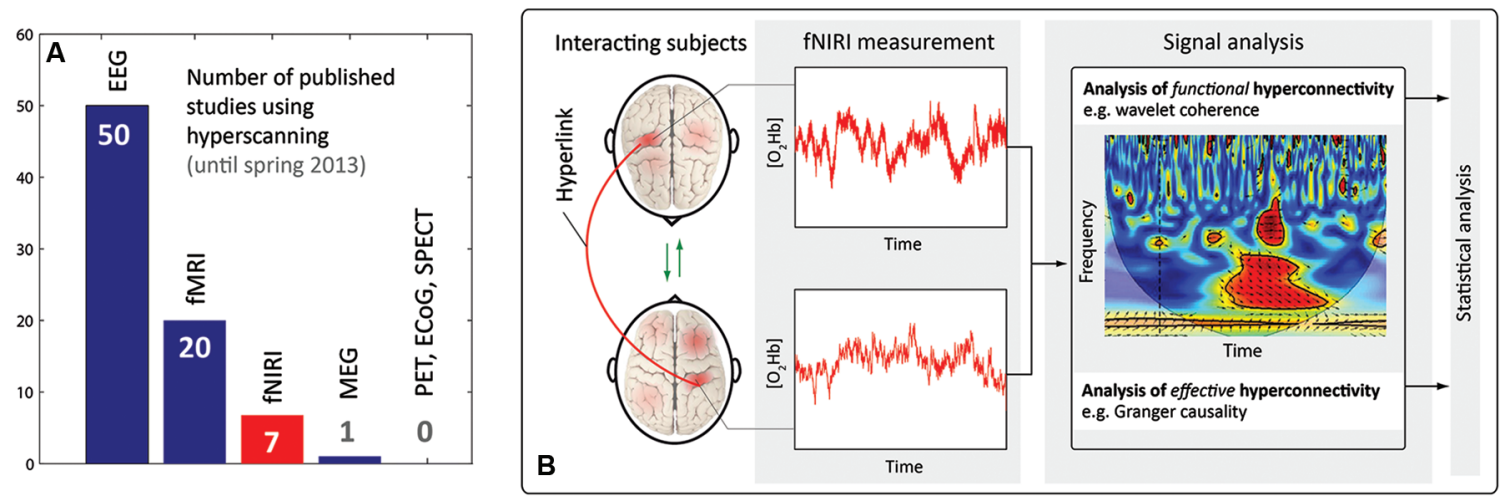

FIGURE 1 [ (A) Number of published hyperscanning studies in the field of neuroscience (according to an own analysis using PubMed and Google Scholar). EEG, fMRI, fNIRI, magneto-encephalography (MEG), positron emission tomography (PET), electrocorticography
(ECOG), single-photon emission computed tomography (SPECT). (B) Visualization of important terms in the context of hyperscanning, and illustration of a typical signal processing for analyzing fNIRI hyperscanning data. 
Table 1 | Listing of fNIRI hyperscanning studies performed so far

\begin{tabular}{lllll}
\hline Reference & \multicolumn{1}{c}{ Task } & fNIRI setup and probe positions & Signal analysis & Results \\
\hline Funane et al. (2011) & Cooperation & 22 ch, R\&L-PFC & Cov & Cov $\uparrow$ \\
Cui et al. (2012) & Cooperation, competition & 22 ch, R\&L-PFC & WC & Coop.: WC $\uparrow$ \\
& & & Comp.: WC - & WC, BA \\
Dommer et al. (2012) & Dual n-back & 4 ch, L-PFC & WC, GC & WC $\uparrow$, GC $\uparrow$ \\
Holper et al. (2012) & Imitation & 4 ch, L-PFC & WC (0.01-0.1 Hz) & Face-to-face communication: WC $\uparrow$ \\
Jiang et al. (2012) & Communication & 20 ch, L-FTPC, 3 ch L-DPFC & Cor & Cor $\uparrow$ \\
Duan et al. (2013) & Competition & 22 ch, L-SMC & BA, Cor & Successful teaching, successful learn- \\
Holper et al. (2013) & Teacher-student & 4 ch, L-PFC & & ing: activity $\downarrow$, Cor $\uparrow$ \\
& interaction & & & \\
\hline
\end{tabular}

Abbreviations: channels (ch), right and left (R\&L), left (L), prefrontal cortex (PFC), inferior parietal lobule (IPL), frontal/temporal/parietal cortices (FTPC), dorsolateral $P F C(D P F C)$, sensorymotor cortex (SMC), covariance (Cov), wavelet coherence (WC), Granger causality (GC), block averaging (BA), correlation (Cor).

two subjects, where either one of the subjects (i.e., the imitator) had to adapt his/her tapping dynamics to the other one (i.e., the model) (imitation task) or both subjects tapped with the same pacing mode (control task). Sixteen subjects participated in the study. [tHb] changes from the left PFC were analyzed and the WC as well as the GC (a measure of the directionality of influence, see also section Opportunities and challenges) was computed. The authors found an increased coherence (in the frequency bands $0.25-0.5 \mathrm{~Hz}$ and $2.5-1 \mathrm{~Hz}$ ) and increased GC during the imitation task. In addition, the causality analysis showed that the cerebral hemodynamics of the imitator adapted to the ones of the model.

Jiang et al. (2012) performed a fNIRI hyperscanning study with 20 subjects performing four different communication tasks, i.e., a face-to-face dialogue, a face-to-face monologue, a backto-back dialogue, and a back-to-back monologue. Changes in $\left[\mathrm{O}_{2} \mathrm{Hb}\right]$ were measured using a multi-channel NIRI device. An optode with 22 channels was placed over the left side of the head so that the frontal, temporal and parietal cortices were covered. Another optode, with 3 channels, was placed above the left DPFC. Synchronization between the brains was determined by calculating the WC (in the frequency band $0.01-0.1 \mathrm{~Hz}$ ). The analysis showed that a coherence increase only occurred during the face-to-face dialogue. The increase was observed over the left inferior frontal cortex.

Duan et al. (2013) implemented a "cross-brain neurofeedback" setup which measured the $\left[\mathrm{O}_{2} \mathrm{Hb}\right]$ changes over the left parietal (sensorimotor) brain in two subjects with a 22 channel fNIRI device while they performed a competitive task ("tug-of-war" game), with feedback information displayed on a screen. The subjects were told to actively imagine that they were physically participating in the tug of war. On the screen, a rope with a ribbon in it was displayed. The aim of the "tug-of-war" game was to pull the ribbon to the left end of the rope (for subject A) or the right end (for subject $\mathrm{B}$ ). The position of the ribbon was controlled by the quotient of $\left[\mathrm{O}_{2} \mathrm{Hb}\right]$ changes from subject $\mathrm{A}$ and $\mathrm{B}$. The online data analysis showed that the subjects were able to control the ribbon position by their brain activity measured online by fNIRI. In an offline analysis, the authors found a decrease in the correlation of the $\left[\mathrm{O}_{2} \mathrm{Hb}\right]$ changes (calculated by the Pearson correlation coefficient) from subject $\mathrm{A}$ and $\mathrm{B}$ when one subject was winning the game, compared to when victory or defeat was not clear. The most recent study was conducted by Holper et al. (2013), employing the same fNIRI-setup as in Dommer et al. (2012). On 17 pairs of subjects, an inter-personal educational dialog task was performed in which subjects performed as teacher-student pairs. For the statistical analysis, both block-averaged hemodynamic activities of $\left[\mathrm{O}_{2} \mathrm{Hb}\right]$ and $[\mathrm{HHb}]$ were measured on the left PFC and the correlation between the teachers' and students' hemodynamic signals was investigated. The analysis revealed that students who successfully acquired knowledge during the dialog had a decreased $\left[\mathrm{O}_{2} \mathrm{Hb}\right]$ during the learning phase compared to the others who did not show a transfer of knowledge. The study further demonstrated that teachers and students showed a positive correlation of cerebral hemodynamic activity when the teaching was successful.

In summary, despite the fact that different experimental paradigms, measurement locations and signal analysis methods have been used, in all of the seven summarized fNIRI hyperscanning studies an inter-personal brain-to-brain coupling was demonstrated. Concerning the measurement position in general, the PFC is of particular interest since it has a role in social interaction and particularly in brain-to-brain coupling (Sänger et al., 2011). Further, whereas in two of the studies the brain activity was measured in the left and right cortices (Funane et al., 2011; Cui et al., 2012), in the other five (Dommer et al., 2012; Holper et al., 2012; Jiang et al., 2012; Duan et al., 2013; Holper et al., 2013) it was measured only in regions in the left part of the brain. One the one hand, the restriction of only measuring regions positioned on the left seems to be justified since it is known, for example, that the centers for perceiving and interpreting social information have been associated with increased activity in the left inferior frontal cortex (Pobric and Hamilton, 2006; Keuken et al., 2011). On the other hand, "visual and motor components of the human mirror system are not left-lateralized" (Aziz-Zadeh et al., 2006) and the right temporal parietal junction is involved in "complex social and moral reasoning" (Miller et al., 2010), highlighting the need to measure both cortices in fNIRI hyperscanning experiments. The observed change in coherence in the LFO range observed by several studies can be either explained by a coupling of the autonomic nervous systems since the LFO amplitude changes reflect primary the vasomotor tone of arterial blood vessels modulated by the sympathetic nervous system (Julien, 2006), or by a local modulation of the neuro-vascular coupling due to neural activity. 


\section{OPPORTUNITIES AND CHALLENGES}

fNIRI hyperscanning bears a great potential for future neuroscience studies since-compared to many other neuroimaging modalities-it offers a cost-effective, easy to apply and reliable technology to measure inter-personal interactions in a more natural context.

One important issue in hyperscanning studies concerns the type of signal processing methods to assess the brain-to-brain coupling. From a neurophysiological point of view, one should distinguish between two types of coupling: functional and effective hyperconnectivity - in analogy to the functional and effective connectivity typically assessed within one brain (Friston, 1994). Functional (hyper-) connectivity refers to a statistical dependence between variables and can be quantified for example by determining the Cor, the correlation (which is a normalized Cov) or the phase-locking of coherence. Effective (hyper-) connectivity refers to a directed causal interaction which can be determined for example using GC or transfer entropy. From a technical point of view, the signal processing methods for analysis of functional and effective hyperconnectivity can be classified according to methods performed in the (i) time, (ii) frequency, or (iii) time-frequency domain. The WC methodology applied in four of the fNIRI studies (Cui et al., 2012; Dommer et al., 2012; Holper et al., 2012; Jiang et al., 2012) is part of the last mentioned class (iii). Figure 1B sketches a typical fNIRI hyperscanning signal processing in the time-frequency domain. The various signal processing methods developed so far for correlation and causality analysis should be exploited in future studies.

Another important issue concerns the experimental paradigms for hyperscanning studies. As summarized by Babiloni and Astolfi (2012), the paradigms employed so far comprise simple motor tasks (e.g., button pressing), music production, interacting by gesticulation, facial expressions, eye contact, verbal dialogue, synchronizing hand or finger movements, or letting the subjects interact in a game theory context. Creating further paradigms that allow optimum capturing of brain-to-brain coupling is an important task for future studies. One difficulty of tasks that involve rhythmic actions (e.g., button pressing) is that they also elicit rhythmic brain activity, which could be misinterpreted as brainto-brain coupling. In order to distinguish between a synchronous brain activity due to the task and a brain-to-brain coupling due to real social interaction, it would be of particular importance to assess the effective hyperconnetivity, and-most importantly-to use appropriate experimental paradigms with appropriate control conditions. The role of brain-to-brain coupling as a function supporting social interaction beyond the coupling in sensorimotor signals between two people remains to be seen.

In addition, a promising option for future hyperscanning studies is applying different modalities simultaneously, e.g., the combined application of fNIRI with fMRI or EEG, or the combination of fNIRI with the measurement of systemic parameters, e.g., HR, electrodermal activity or changes in respiration. Also the continuous measurement of the blood pressure and arterial $\mathrm{CO}_{2}$ may be important to exclude confounding factors in fNIRI measurements, as highlighted recently by Tachtsidis et al. (2009) and Scholkmann et al. (2013a), respectively. The measurement of systemic parameters during hyperscanning studies is not only important in order to exclude confounders but also to elucidate the mechanisms enabling the brain-to-brain coupling. What is known so far is that the interaction between persons includes neuronal and systemic physiological processes, leading to a coupling of not only brain states but also states of the whole physiology, mainly happening unconsciously. Examples for this are the increase in breathing of a person that observes exertion (e.g., weight lifting) (Paccalin and Jeannerod, 2000), postural responses when observing human imbalance (Tia et al., 2011), posture and body movement synchronization (Bernieri, 1988; Chartrand and Bargh, 1999; Sharpley et al., 2001; Yun et al., 2012), and synchronization of HR and respiration (Florian et al., 1998; Mcfarland, 2001; Konvalinka et al., 2011; Xygalatas et al., 2011) phenomena that are known as mimicry, automatic imitation (e.g., postural responses due to observation) and entrainment (e.g., posture/body and HR/respiration synchronization; Knoblich and Sebanz, 2008; Chartrand and Van Baaren, 2009; Heynes, 2011; Kinsbourne and Helt, 2011).

To improve the sensitivity of the fNIRI measurement to cerebral hemodynamics and oxygenation it would be desirable for future studies to apply methods that reduce the influence of superficial changes on the measured signal. Such methods comprise hardware (e.g., Hueber et al., 1999; Suzuki et al., 1999) or signal processing approaches (e.g., Saager and Berger, 2005). Also the analysis of changes in $[\mathrm{HHb}],\left[\mathrm{O}_{2} \mathrm{Hb}\right]$ and $[\mathrm{tHb}]$, and not only in one signal alone (i.e., $\left[\mathrm{O}_{2} \mathrm{Hb}\right]$ or $[\mathrm{tHb}]$ ), will help to distinguish between systematically and neuronally driven changes.

An inherent limitation of fNIRI is that only cortical brain regions can be accessed. The method is not able to measure subcortical areas. However, the fact that important brain regions for social interaction are located in the cerebral cortex makes this limitation less significant.

\section{CONCLUSION}

fNIRI hyperscanning is a promising new field in social neuroscience with a great potential to gain further insights into the neurobiological correlates of inter-personal interactions. fNIRI studies performed so far using this methodological approach are promising and demonstrated the feasibility of fNIRI for hyperscanning. We suggest for future studies (i) to exploit the variety of signal processing methods already available for quantifying the between-brain coupling and improving the signal quality, and (ii) to realize multi-modal fNIRI hyperscanning measurements by combining fNIRI with other neuroimaging or physiological measurements.

\section{ACKNOWLEDGMENTS}

We thank Rachel Scholkmann for proofreading of the manuscript and Raphael Zimmermann, Dr. Xu Cui and Prof. Karl J. Friston for the fruitful discussions.

\section{REFERENCES}

Astolfi, L., Toppi, J., Fallani, F. D. V., Vecchiato, G., Cincotti, F., Wilke, C. T., et al. (2011). Imaging the social brain by simultaneous hyperscanning during subject interaction. IEEE Intell. Syst. 26, 38-45. doi: 10.1109/mis.2011.61 
Aziz-Zadeh, L., Koski, L., Zaidel, E., Mazziotta, J., and Iocoboni, M. (2006). Lateralization of the human mirror neuron system. J. Neurosci. 26, 2964-2970. doi: 10.1523/jneurosci.2921-05.2006

Babiloni, F., and Astolfi, L. (2012). Social neuroscience and hyperscanning techniques: past, present and future. Neurosci. Biobehav. Rev. doi: 10.1016/j.neubiorev.2012.07.006. [Epub ahead of print].

Bernhardt, B. C., and Singer, T. (2012). The neural basis of empathy. Annu. Rev. Neurosci. 35, 1-23. doi: 10.1146/annurev-neuro-062111-150536

Bernieri, F. J. (1988). Coordinated movement and rapport in teacher-student interactions. J. Nonverbal Behav. 12, 120-138. doi: 10.1007/bf00986930

Cacioppo, J. T., and Berntson, G. G. (1992). Social psychological contributions to the decade of the brain. Doctrine of multilevel analysis. Am. Psychol. 47, 10191028. doi: 10.1037/0003-066x.47.8.1019

Chartrand, T. L., and Bargh, J. A. (1999). The chameleon effect: the perceptionbehavior link and social interaction. J. Pers. Soc. Psychol. 76, 893-910. doi: 10. 1037//0022-3514.76.6.893

Chartrand, T. L., and Van Baaren, R. (2009). Human mimicry. Adv. Exp. Soc. Psychol. 41, 219-274. doi: 10.1016/S0065-2601(08)00405-X

Chatel-Goldman, J., Schwartz, J. L., Jutten, C., and Congedo, M. (2013). Non-local mind from the perspective of social cognition. Front. Hum. Neurosci. 7:107. doi: 10.3389/fnhum.2013.00107

Cui, X., Bryant, D. M., and Reiss, A. L. (2012). NIRS-based hyperscanning reveals increased interpersonal coherence in superior frontal cortex during cooperation. Neuroimage 59, 2430-2437. doi: 10.1016/j.neuroimage.2011. 09.003

Dommer, L., Jäger, N., Scholkmann, F., Wolf, M., and Holper, L. (2012). Betweenbrain coherence during joint n-back task performance: a two-person functional near-infrared spectroscopy study. Behav. Brain Res. 234, 212-222. doi: 10.1016/j. bbr.2012.06.024

Duan, L., Liu, W.-J., Dai, R.-N., Li, R., Lu, C.-M., and Huang, Y.-X. (2013). Crossbrain neurofeedback: scientific concept and experimental platform. PLoS One 8:e64590. doi: 10.1371/journal.pone.0064590

Duane, T. D., and Behrendt, T. (1965). Extrasensory electroencephalographic induction between identical twins. Science 150:367. doi: 10.1126/science.150. 3694.367

Dumas, G., Lachat, F., Martinerie, J., Nadel, J., and George, N. (2011). From social behaviour to brain synchronization: review and perspectives in hyperscanning. IRBM 32, 48-53. doi: 10.1016/j.irbm.2011.01.002

Ferrari, M., and Quaresima, V. (2012). A brief review on the history of human functional near-infrared spectroscopy (fNIRS) development and fields of application. Neuroimage 63, 921-935. doi: 10.1016/j.neuroimage.2012. 03.049

Florian, G., Stancák, A., and Pfurtscheller, G. (1998). Cardiac response induced by voluntary self-paced finger movement. Int. J. Psychophysiol. 28, 273-283. doi: 10. 1016/s0167-8760(97)00075-5

Friston, K. J. (1994). Functional and effective connectivity in neuroimaging: a synthesis. Hum. Brain Mapp. 2, 56-78. doi: 10.1002/hbm.460020107

Frith, C. D. (2007). The social brain? Philos. Trans. R. Soc. Lond. B Biol. Sci. 362, 671-678. doi: 10.1098/rstb.2006.2003

Frith, U., and Frith, C. (2001). The biological basis of social interaction. Curr. Dir. Psychol. Sci. 10, 151-155. doi: 10.1111/1467-8721.00137

Froese, T., Gershenson, C., and Rosenblueth, D. A. (2013). "The dynamically extended mind," in IEEE Congress on Evolutionary Computation (CEC), 14191426. doi: 10.1109/CEC.2013.6557730

Funane, T., Kiguchi, M., Atsumori, H., Sato, H., Kubota, K., and Koizumi, H. (2011). Synchronous activity of two people's prefrontal cortices during a cooperative task measured by simultaneous near-infrared spectroscopy. J. Biomed. Opt. 16:077011. doi: 10.1117/1.3602853

Genvins, A., Chan, C. S., and Sam-Vargas, L. (2012). Towards measuring brain function on groups of people in the real world. PLoS One 7:e44676. doi: 10. 1371/journal.pone.0044676

Hari, R., and Kujala, M. V. (2009). Brain basis of human social interaction: from concepts to brain imaging. Physiol. Rev. 89, 453-479. doi: 10.1152/physrev. 00041.2007

Hasson, U., Ghazanfar, A. A., Galantucci, B., Garrod, S., and Keysers, C. (2012). Brain-to-brain coupling: a mechanism for creating and sharing a social world. Trends Cogn. Sci. 16, 114-121. doi: 10.1016/j.tics.2011.12.007
Hearne, K. (1977). Visually evoked responses and ESP: an experiment. J. Soc. Psych. Res. 49, 648-657.

Heynes, C. (2011). Automatic imitation. Psychol. Bull. 137, 463-483. doi: 10. 1037/a0022288

Holper, L., Goldin, A. P., Shalóm, D. E., Battro, A. M., Wolf, M., and Sigman, M. (2013). The teaching and the learning brain: a cortical hemodynamic marker of teacher-student interactions in the Socratic dialog. Int. J. Educ. Res. 59, 1-10. doi: 10.1016/j.ijer.2013.02.002

Holper, L., Scholkmann, F., and Wolf, M. (2012). Between-brain connectivity during imitation measured by fNIRS. Neuroimage 63, 212-222. doi: 10.1016/j. neuroimage.2012.06.028

Hueber, D. M., Fantini, S., Cerussi, A. E., and Barbieri, B. B. (1999). New optical probe designs for absolute (self-calibrating) NIR tissue hemoglobin measurements. Opt. Tomogr. Spectrosc. Tissue III: Proc. SPIE 3597, 618-631. doi: 10. $1117 / 12.356784$

Jiang, J., Dai, B., Peng, D., Zhu, C., Liu, L., and Lu, C. (2012). Neural synchronization during face-to-face communication. J. Neurosci. 32, 16064-16069. doi: 10. 1523/jneurosci.2926-12.2012

Julien, C. (2006). The enigma of mayer waves: facts and models. Cardiovasc. Res. 70, 12-21. doi: 10.1016/j.cardiores.2005.11.008

Keuken, M. C., Hardie, A., Dorn, B. T., Dev, S., Paulus, M. P., Jonas, K. J., et al. (2011). The role of the left inferior frontal gyrus in social perception: an rTMS study. Brain Res. 1383, 196-205. doi: 10.1016/j.brainres.2011.01. 073

Kinsbourne, M., and Helt, M. (2011). "Entrainment, mimicry and interpersonal synchrony," in The Neurophysiology of Autism, ed D. Fein (Oxford, New York: Oxford University Press), 339-365.

Knoblich, G., and Sebanz, N. (2008). Evolving intentions for social interaction: from entrainment to joint action. Philos. Trans. R. Soc. Lond. B Biol. Sci. 363, 2021-2031. doi: 10.1098/rstb.2008.0006

Konvalinka, I., and Roepstorff, A. (2012). The two-brain approach: how can mutually interacting brains teach us something about social interaction? Front. Hum. Neurosci. 6:215. doi: 10.3389/fnhum.2012.00215

Konvalinka, I., Xygalatas, D., Bulbulia, J., Schjødt, U., Jegindø, E.-M., Wallrot, S., et al. (2011). Synchronized arousal between performers and related spectators in a fire-walking ritual. Proc. Natl. Acad. Sci. U S A 108, 8514-8519. doi: 10. 1073/pnas.1016955108

Mcfarland, D. H. (2001). Respiratory markers of conversational interaction J. Speech Lang. Hear. Res. 44, 128-143. doi: 10.1044/1092-4388(2001/012)

Miller, M. B., Sinnott-Armstrong, W., Young, L., King, D., Paggi, A., Fabri, M., et al. (2010). Abnormal moral reasoning in complete and partial callosotomy patients. Neuropsychologia 48, 2215-2220. doi: 10.1016/j.neuropsychologia. 2010.02.021

Montague, P. R., Berns, G. S., Cohen, J. D., Mcclure, S. M., Pagnoni, G., Dhamala, M., et al. (2002). Hyperscanning: simultaneous fMRI during linked social interactions. Neuroimage 16, 1159-1164. doi: 10.1006/nimg.2002. 1150

Muehlemann, T., Haensse, D., and Wolf, M. (2008). Wireless miniaturized invivo near infrared imaging. Opt. Express 16, 10323-10330. doi: 10.1364/oe.16. 010323

Paccalin, C., and Jeannerod, M. (2000). Changes in breathing during observation of effortful actions. Brain Res. 862, 194-200. doi: 10.1016/s0006-8993(00)02145-4

Pobric, G., and Hamilton, A. F. (2006). Action understanding requires the left inferior frontal cortex. Curr. Biol. 16, 524-529. doi: 10.1016/j.cub.2006.01.033

Premack, D., and Woodruff, G. (1978). Does the chimpanzee have a theory of mind? Behav. Brain Sci. 1, 515-526. doi: 10.1017/s0140525x00076512

Przyrembel, M., Smallwood, J., Pauen, M., and Singer, T. (2012). Illuminating the dark matter of social neuroscience: considering the problem of social interaction from philosophical, psychological and neuroscientific perspectives. Front. Hum. Neurosci. 6:190. doi: 10.3389/fnhum.2012.00190

Saager, R. B., and Berger, A. J. (2005). Direct characterization and removal of interfering absorption trends in two-layer turbid media. J. Opt. Soc. Am. A Opt. Image Sci. Vis. 22, 1874-1882. doi: 10.1364/josaa.22.001874

Sänger, J., Lindenberger, U., and Müller, V. (2011). Interactive brain, social minds. Commun. Integr. Biol. 4, 655-663.

Saxe, R. (2006). Uniquely human social cognition. Curr. Opin. Neurobiol. 16, $235-$ 239. doi: 10.1016/j.conb.2006.03.001 
Schilbach, L., Timmermans, B., Reddy, V., Costall, A., Bente, G., Schlicht, T., et al. (2013). Toward a second-person neuroscience. Behav. Brain Sci. 36, 393-414. doi: 10.1017/s0140525x1200204x

Scholkmann, F., Gerber, U., Wolf, M., and Wolf, U. (2013a). End-tidal $\mathrm{CO}_{2}$ : an important parameter for a correct interpretation in functional brain studies using speech tasks. Neuroimage doi: 10.1016/j.neuroimage.2012.10.025. [Epub ahead of print].

Scholkmann, F., Kleiser, S., Metz, A., Zimmermann, R., Mata Pavia, J., Wolf, U., et al. (2013b). A review on continuous wave functional near-infrared spectroscopy and imaging instrumentation and methodology. Neuroimage doi: 10.1016/j.neuroimage.2013.05.004. [Epub ahead of print].

Sharpley, C. F., Halat, J., Rabinowicz, T., Weiland, B., and Stafford, J. (2001). Standard posture, postural mirroring and client-perceived rapport. Couns. Psychol. Q. 14, 267-280. doi: 10.1080/09515070110088843

Suzuki, S., Takasaki, S., Ozaki, T., and Kobayashi, Y. (1999). A tissue oxygenation monitor using NIR spatially resolved spectroscopy. Proc. SPIE 3597, 582-592. doi: $10.1117 / 12.356862$

Tachtsidis, I., Leung, T. S., Chopra, A., Koh, P. H., Reid, C. B., and Elwell, C. E. (2009). False positives in functional nearinfrared topography. Adv. Exp. Med. Biol. 645, 307-314. doi: 10.1007/978-0-387-85998-9_46

Tia, B., Saimpont, A., Paizis, C., Mourey, F., Fadiga, L., and Pozzo, T. (2011). Does observation of postural imbalance induce a postural reaction? PLoS One 6:e17799. doi: 10.1371/journal.pone.0017799

Watanabe, K. (2013). Teaching as a dynamic phenomenon with interpersonal interactions. Mind Brain Educ. 7, 91-100. doi: 10.1111/mbe.12011
Xygalatas, D., Konvalinka, I., Bulbulia, J., and Roepstorff, A. (2011). Quantifying collective effervescence: Heart-rate dynamics at a fire-walking ritual. Commun. Integr. Biol. 4, 735-738.

Yun, K., Watanabe, K., and Shimojo, S. (2012). Interpersonal body and neural synchronization as a marker of implicit social interaction. Sci. Rep. 2:959. doi: $10.1038 /$ srep00959

Conflict of Interest Statement: The authors declare that the research was conducted in the absence of any commercial or financial relationships that could be construed as a potential conflict of interest.

Received: 08 August 2013; accepted: 10 November 2013; published online: 27 November 2013.

Citation: Scholkmann F, Holper L, Wolf $U$ and Wolf M (2013) A new methodical approach in neuroscience: assessing inter-personal brain coupling using functional near-infrared imaging (fNIRI) hyperscanning. Front. Hum. Neurosci. 7:813. doi: 10.3389/fnhum.2013.00813

This article was submitted to the journal Frontiers in Human Neuroscience.

Copyright (C) 2013 Scholkmann, Holper, Wolf and Wolf. This is an open-access article distributed under the terms of the Creative Commons Attribution License (CC BY). The use, distribution or reproduction in other forums is permitted, provided the original author(s) or licensor are credited and that the original publication in this journal is cited, in accordance with accepted academic practice. No use, distribution or reproduction is permitted which does not comply with these terms. 